\title{
Comparative study between Silodosin alone and Silodosin plus Tadalafil for the medical management of lower Ureteric Stone in South-Western part of the Bangladesh
}

\author{
Masud Ahmed ${ }^{1}$, Prodyut Kumar Saha ${ }^{2}$, Kartik Chandra Ghosh ${ }^{3}$, Sk. Amirul Islam ${ }^{4}$, Nirupom Mondal ${ }^{5}$
}

Received: 06 - 06 - 2020

Accepted: $17-08-2020$

Conflicts of interest: None

Keywords: Silodosin, Tadalafil, Ureteric calculi. Medical Expuslsion Therapy.

\begin{abstract}
Objective: To compare the treatment outcome of Silodosin alone and Silodosin plus Tadalafil as a medical expulsive therapy (MET) of lower ureteric stone in south-western part of Bangladesh.

Methodology: The study was conducted in a tertiary hospital in Khulna, over a period of 12 months (January 2019 to December 2019). Out of 108 patients, 100 meet the inclusion criteria who were purposively assigned into 2 groups. 48 patients included in Silodosin alone group and 52 in Silodosin plus Tadalafil group.
\end{abstract}

Result: There was a significant higher stone expulsion rate in Silodosin plus Tadalafil than Silodosin alone which was $88.46 \%$ vs $75 \%$ respectively (P value 0.02). The mean stone expulsion time of Silodosin alone was14.33 ( \pm 3.1 ) days and Silodosin plus Tadalafil was 11.48( \pm 2.3$)$ days (P value 0.001). The episodes of pain in Silodosin alone were $0.7( \pm 0.06)$ and $0.6( \pm 0.2)$ in Silodosin plus Tadalafil group that was statistically significant.

Conclusion: The present study concludes that Silodosin plus tadalafil combination therapy significantly increases ureteric stone expulsion rate and decreases the expulsion time and pain episodes than treatment with silodosin alone.

\section{Introduction}

Urolithiasis is one of the common diseases and affects $5-10 \%$ of people globally. Renal stones are most prevalent between the ages of 20 and 40 years and are three times more common in men than women. $20 \%$ of all urinary stones are located in the ureter. Out of them, $2 / 3^{\text {rd }}$ stones present in lower ureter. Medical expulsive therapy (MET) is one of the routine treatment options for small lower ureteric stones, and the therapy uses various drugs acting on the ureteric smooth muscle by different mechanism. There has been a steep rise of minimally invasive procedures for ureteric stones as well as renal stones management. ${ }^{1,2,3}$ However, medical expulsive therapy is still regarded as an established treatment option for the management of distal ureteric stones (DUS). Stone location, size, number, ureteric spasm, mucosal edema or inflammation and ureteric anatomy are the key factors that influence the passage of ureteric stones. ${ }^{4}$ Ureteroscopy and shock wave lithotripsy (SWL) remain the most effective treatments for DUS; however, they are expensive and not risk free. Spontaneous stone

1 Assistant Professor, Department of Urology, Khulna Medical College, Khulna

2 Associate Professor, Department of Urology, Dhaka Medical College, Dhaka.

3. Assistant Professor, Department of Urology, Bangabandhu Sheikh Mujib Medical University, Dhaka.

4. Registrar (Uro-oncology), Department of Urology, National Institute of Cancer Research \& Hospital, Dhaka.

5. Registrar, Department of Urology, Khulna Medical College, Khulna

Correspondence: Dr. Masud Ahmed, Assistant Professor, Department of Urology, Khulna Medical College, Khulna, E-mail: uromasud@gmail.com 
expulsion can occur in up to $50 \%$ of cases of stone less than $6 \mathrm{~mm}$. Nevertheless, many complications such as ureteric colic, UTI, and hydronephrosis may occur. Recently, the use of various drugs as MET for DUS has escalated the rate of stone clearance and reduced complications. Silodosin is a more selective á1Aadrenergic receptor antagonist and has a better stone expulsion rate than tamsulosin. ${ }^{5}$ Tadalafil, a PDE- 5 inhibitor either alone or combined with tamsulosin is safe, efficacious and well tolerated for the treatment of lower ureteric stones. ${ }^{6}$ Tadalafilhas replaced sildenafil due to less visual problems and its absorption does not appear to be affected by meals. ${ }^{7}$ The combination of silodosin and tadalafil has greater potency than either drug alone for the treatment of LUTS associated with BPH, but no study has been reported using these two drugs in combination for the treatment of lower ureteric stones in this region.

\section{Methodology:}

This prospective study (Quasi experimental) was conducted at Khulna Medical College Hospital, a tertiary Hospital in this region and few private hospitals in Khulna over a period of 12 months (January 2019 to December 2019). All patients with lower ureteric stone from $5 \mathrm{~mm}$ to $10 \mathrm{~mm}$ in size, diagnosed by non-contrast CT Scan, Ultrasound (USG) of KUB or IVU/ X-ray KUB and given inform written consent were only included in the study. CT scan was not done in all patients due to financial reason. Patients with the presence of multiple ureteric stones, radiolucent stones, urinary tract infection, pregnancy, pediatric population and history of ureteral surgery or previous endoscopic procedures and who did not given written consent were excluded in this study. The exclusion criteria also extended with patients having ischemic heart disease, congestive cardiac failure, or complicated hypertension, raised serum creatinine and those requiring emergency intervention. Patients were purposively divided into two groups in Group A patient who taken only Silodosin alone and in Group B patient who taken Silodosin plus Tadalafil. Out of 108 patients, 100 meet the inclusion criteria. Five patients from Group A (Silodosin alone) and three patients from Group B (Silodosin plus Tadalafil) dropped out during follow up for various reasons. Finally, we included 48 patients in Group A (Silodosin alone) and 52 in Group B (Silodosin plus Tadalafil). Group A was given silodosin $8 \mathrm{mg}$ once daily and Group B was given silodosin $8 \mathrm{mg}$ plus Tadalafil $5 \mathrm{mg}$ once daily. In both groups, drugs were continued till stone expulsion, but not more than 3 weeks. There was no strong evidence that the long duration use of these drugs will increase the expulsion rate orminimize the deleterious effect of obstructive uropathy. Patients were instructed to drink plenty of fluids and take one tablet of diclofenac $50 \mathrm{mg}$ orally during episode of pain with a maximum dose of $150 \mathrm{mg}$ per day ${ }^{2}$. Patients who either could not present the stone or present the stone that did not match the original size and shape were evaluated by physical examination, serum creatinine and the same imagings by which lower ureteric stones were conformed previously. In case of doubt, NCCT KUB was done despite previous imaging modality to conform stone expulsion. Expulsion of the ureteric calculi, total dose of analgesic used, number of colic episodes and emergency room visits, and side effect of drugs were recorded. Semi-rigid ureteroscopy was done to those who did not pass stones after 3 weeks of follow-up for stone removal. Unpaired Student's $t$-test and the $\div 2$-test were used for the analysis of the variables and categorical data. Differences were considered significant at a $P$ value less than 0.05 .

\section{Results:}

Table-I shows that baseline characteristics of mean age was 36.25( \pm 11.17$)$ years in Silodosin alone group and $37.38( \pm 12.10)$ years in Silodosin plus Tadalafil group, mean BMI $(\mathrm{kg} / \mathrm{m} 2) 23.65( \pm 4.16)$ was in Silodosin alone group and $24.12( \pm 5.12)$ was in Silodosin plus Tadalafil group, mean stone size $7.6( \pm 1.25) \mathrm{mm}$ in Silodosin alone group and $7.7( \pm 1.30) \mathrm{mm}$ in Silodosin plus Tadalafil group. Sex, age, BMI, stone size and side of stone were not statistically significant $(p>0.05)$ between two groups. Table-II shows higher stone expulsion rate in Silodosin plus Tadalafil than Silodosin alone which was $88.46 \%$ vs $75 \%$ respectively ( $P$ value 0.02 )that was statistically significant. The mean expulsion time Silodosin alone was 14.33( \pm 3.1$)$ and11.48 $( \pm 2.3)$ was in Silodosin plus Tadalafil group $(<0.001)$. The pain episodes $0.7( \pm 0.06)$ were in Silodosin alone and $0.6( \pm 0.2)$ were in Silodosin plus Tadalafil group $(<0.001)$ that were statistically significant (Table-II). Regarding adverse effects, such as headache, dizziness, backache, orthostatic hypotension and retrograde ejaculation were similar in Silodosin alone groups and Silodosin plus Tadalafil Group (Table-III). That was not statistically significant $(\mathrm{P}>0.05)$ 
Table I : Baseline characteristics of the study patients $(n=100)$

\begin{tabular}{lccc}
\hline Baseline characteristics & Silodosin alone $(\mathrm{n}=48)$ & Silodosin plus Tadalafil $(\mathrm{n}=52)$ & P value \\
\hline Male & $27(56.25 \%)$ & $33(63.46 \%)$ & $0.414^{\mathrm{ns}}$ \\
Female & $21(43.75 \%)$ & $19(36.54 \%)$ & \\
Age $($ years $)$ & $36.25( \pm 11.17)$ & $37.38( \pm 12.10)$ & $0.629^{\mathrm{ns}}$ \\
BMI $(\mathrm{kg} / \mathrm{m} 2)$ & $23.65( \pm 4.16)$ & $24.12( \pm 5.12)$ & $0.616^{\mathrm{ns}}$ \\
Stone size $(\mathrm{mm})$ & $7.6( \pm 1.25)$ & $7.7( \pm 1.30)$ & $0.695^{\mathrm{ns}}$ \\
Stone side & & & $.409^{\mathrm{ns}}$ \\
Right & $30(62.5 \%)$ & $31(59.62 \%)$ & \\
Left & $18(37.5 \%)$ & $21(40.38 \%)$ & \\
\hline
\end{tabular}

$\mathrm{Ns}=$ not significant, $\mathrm{P}$ value reached from $\mathrm{Chi}$ square and unpaired $\mathrm{t}$-test

Table II : Treatment outcome $(n=100)$

\begin{tabular}{lccc}
\hline Treatment outcome & Silodosin alone $(\mathrm{n}=48)$ & Silodosin plus Tadalafil $(\mathrm{n}=52)$ & P value \\
\hline Expulsion rate (\%) & $75.0 \%$ & $88.46 \%$ & $0.029^{\mathrm{s}}$ \\
Expulsion time (days) & $14.33( \pm 3.1)$ & $11.48( \pm 3.1)$ & $0.001^{\mathrm{s}}$ \\
Pain episodes & $0.7( \pm 0.06)$ & $0.6( \pm 0.2)$ & $0.001^{\mathrm{s}}$ \\
\hline
\end{tabular}

$\mathrm{s}=$ significant, $\mathrm{P}$ value reached from $\mathrm{Chi}$ square and unpaired $\mathrm{t}$-test

Table III : Distribution of the study patients by side effects $(n=100)$

\begin{tabular}{lccl}
\hline Side effects & Silodosin alone $(\mathrm{n}=48)$ & Silodosin plus Tadalafil $(\mathrm{n}=52)$ & $\mathrm{p}$ value \\
\hline Headache & $5(10.42 \%)$ & $7(13.46 \%)$ & $0.538^{\mathrm{ns}}$ \\
Dizziness & $4(8.33 \%)$ & $5(9.62 \%)$ & $0.822^{\mathrm{ns}}$ \\
Backache & $4(8.33 \%)$ & $5(9.62 \%)$ & $0.822^{\text {ns }}$ \\
Orthostatic hypotension & $3(6.25 \%)$ & $2(3.85 \%)$ & $0.581^{\mathrm{ns}}$ \\
Retrograde ejaculation & $8(16.67 \%)$ & $8(15.38 \%)$ & $0.790^{\text {ns }}$ \\
\hline
\end{tabular}

$\mathrm{Ns}=$ not significant, $\mathrm{P}$ value reached from Chi square test and Fisher's exact test

\section{Discussion}

This study observed the mean age was $36.25( \pm 11.17)$ years inSilodosin alone group and $37.38( \pm 12.10)$ years in Silodosin plus Tadalafil group, mean BMI $(\mathrm{kg} / \mathrm{m} 2)$ $23.65( \pm 4.16)$ was in Silodosin alone group and 24.12 $( \pm 5.12)$ was in Silodosin plus Tadalafil group, mean stone size 7.6( \pm 1.25$) \mathrm{mm}$ in Silodosin alone group and $7.7( \pm 1.30)$ mmin Silodosin plus Tadalafil group. Rahman et al. ${ }^{9}$ reported there was no statistically significant difference between the groups for patient's age, gender, body mass index, or stone size. They observed that the mean age was found $34( \pm 12)$ in
Silodosin alone and $35( \pm 10)$ in Silodosin plus tadalafil group, stone size $7.4( \pm 1.30) \mathrm{mm}$ in Silodosin alone and $7.6( \pm 1.35) \mathrm{mm}$ in Silodosin plus tadalafil group.

Elgalaly et al. ${ }^{5}$ reported that mean age was found 33.6 $( \pm 9.9)$ ingroup A and $35.5( \pm 11.3)$ group B. Similar observation found in Hari Bahadur KC etal. ${ }^{10}$ They reported that the patients' mean age was $31.72 \pm 12.63$ years (range, 18-68 years) and the male to female ratio was 1.5 . The mean stone size was $7.09 \pm 1.2$ and $7.13 \pm 1.5 \mathrm{~mm}$ in groups $\mathrm{A}$ and $\mathrm{B}$, respectively.

Many factors influencing the spontaneous expulsion of stones, such as stone location, stone size, stone 
number, stone structure, ureteral spasm, mucosal edema or inflammation and ureteral anatomy. Therefore, the use of MET is justifiable to reduce edema and spasm and relax the smooth muscles for stone expulsion. The American Urological Association (AUA) as well as the European Urological Association (EUA) ureteric stones clinical guidelines support the use of MET for patients with distal ureteral calculi of $<10 \mathrm{~mm}$. In comparison with surgical intervention for ureteric stones, MET has a high safety profile and very low cost ${ }^{9}$.

Jayant et al reported that a combination of tadalafil with tamsulosin had better outcomes in ureteric stone expulsion. In their study, the stone expulsion ratewas 83.6\% $(\mathrm{P}=0.031)$. In our present study, Group B (Silodosin plus Tadalafil)had a significant higher stone expulsion rate $(88.46 \%)$ compared to Silodosin alone $(\mathrm{P}=0.029)^{11}$.

Highly selective á1A-adrenoceptor blockers have been developed to minimize the cardiovascular adverse effects while maintaining their efficacy on the urinary tract.Tamsulosin is a selective á1-blocker with a 10-fold greater affinity for the á1A- adrenoceptor than for á1Badrenoceptor subtype, while the affinity of silodosin for the á1A-adrenoceptor is50-fold greater which explains the weak cardiovascular adverse effects of silodosin $^{15}$. For this reason, Silodosin is better than Tamsulosin.

In current study observed that Silodosin plus Tadalafil showed a higher stone expulsion rate than Silodosin alone which was $88.46 \%$ vs $75 \%$ respectively ( $P$ value $0.02)$ that was statistically significant. The mean expulsion time Silodosin alone was $14.33( \pm 3.1)$ and $11.48( \pm 2.3)$ was in Silodosin plus Tadalafil group.

This study showed, the episode of pain was $0.7( \pm 0.06)$ in Silodosin alone and 0.6 $( \pm 0.2)$ in Silodosin plus Tadalafil group $(<0.001)$ that was statistically significant. In Rahman et al. ${ }^{9}$ study, Silodosin+tadalafil Group had a significantly higher stone expulsion rate $(90 \%)$ compared to Silodosin alone groups. They observed that the mean stone expulsion time in Silodosin plus tadalafil Group was also significantly less (12 \pm 2.2$)$ days compared to Silodosin alone Group $(15 \pm 3.3)$ days $(P<0.001)$. In the Jayant etal. study ${ }^{11}$, the mean expulsion time was $14.9( \pm 4.4)$ days with the tadalafil and tamsulosin combination compared to 16.7
(4.8) days for tamsulosin alone ( $\mathrm{P}=0.003$ ).In Rahman et al. ${ }^{9}$ study, tadalafil and tamsulosin Group had significantly fewer pain episodes than Silodosin alone Group $(\mathrm{P}<0.001)$.

Jayantetal. ${ }^{11}$ also showed significant lower pain episodes with a tadalafil and tamsulosin combination compared to tamsulosin alone. This may be due to two drugs with different actions on the ureter. The stone expulsion rate was $79.0 \%$ in Group A and $62.5 \%$ in Group $\mathrm{B}($ Pvalue $=0.025)$. The mean time for stone expulsion in Group A was 1.66 vs. 2.32 weeks in GroupB $(P$ value $=0.001)$. Combination of tamsulosin and tadalafil was found to be safe by Kloneret al. ${ }^{12}$ Bechara etal. ${ }^{13}$ showed effectiveness of combination when they used for LUTS. ${ }^{14}$

While comparing the efficacy of drugs in Gnyawaliet al. ${ }^{1}$ study, they found Group A(tamsulosin plus tadalafil) patients had higher expulsion rate than Group B (tamsulosin) $79.01 \%$ vs. $62.50 \%$ ( $P$ value 0.025$)$ respectively. Hari Bahadur KC et al. ${ }^{10}$ reported that the overall stone expulsion rate in the study was $72.9 \%$ (62 of 85). The stone expulsion rate was significantly higher in group B than in group A $(84.1 \%$ vs. $61.0 \%$, $\mathrm{p}=0.017)$. The mean stone expulsion time was lower in group B ( $8.08 \pm 3.3$ days) than in group A $(9.64 \pm 3.8$ days), but this difference was not significant $(\mathrm{p}=0.094)$.

Regarding adverse effects, such as headache, dizziness, backache, orthostatic hypotension and retrograde ejaculation were a little bit higher in Silodosin plus Tadalafil Group than Silodosin alone; however, it was not statistically significant $(\mathrm{P}>0.05)$. No serious sideeffects were encountered in the Rahman et al. ${ }^{9}$ study. In Gnyawaliet al. ${ }^{1}$ study shows drug-related adverse effects such as headache, dizziness, postural hypotension, backache, and runny nose were comparable between two groups and not statistically significant.

\section{Conclusion}

The present study concludes that combination of silodosin and tadalafil increases the ureteric stone expulsion rate, decreases stone expulsion time and reduces episode of pain significantly than silodosin alone without any serious side-effects.

\section{Limitation}

Small group study and not randomized.

\section{Recommendation}

Large number of sample and multi-centered double blind randomized control trail should be taken. 


\section{References}

1. Gnyawali D, Man Pradhan M, Raj SigdelP ,Parajuli P, Chudal S, Poudyal S, et al.Efficacy of Tamsulosin plus Tadalafil versus Tamsulosin as Medical Expulsive Therapyfor Lower Ureteric Stones: A Randomized Controlled Trial. Advances in Urology,2020;Article ID 4347598:1-5

2. Puvvada S, Mylarappa P, Aggarwal K, Patil A, Joshi P and Desigowda R. "Comparativeefficacy of tadalafil versus tamsulosin as the medical expulsive therapy in lower uretericstone: a prospective randomized trial," Central European Journal of Urology,2016;69(69):178-182.

3. Pietropaolo A, Proietti S, Geraghty R, Skolarikos A, Papatsoris E, Liatsikos E, et al.Trends of 'urolithiasis: interventions simulation, and laser technology' over the last 16years (2000-2015) as published in the literature (PubMed): a systematic review fromEuropean section of Uro-technology (ESUT). World J Urol 2017;35:1651-8.

4. Wein AJ, Kavoussi LR, Partin AW, Peters CA. Campbell-Walsh urology. 11th ed.Philadelphia: Elsevier; 2016. p. 3743.

5. Elgalaly H, Sakr A, Fawzi A, A. Salem E, Desoky E, Shahin A \&Kamel M. Silodosin vstamsulosin in the management of distal ureteric stones: A prospective randomisedstudy,Arab Journal of Urology,2016;14:1:12-17

6. Bai Y, Yang Y, Wang X, Tang Y, Han P, Wang J. Tadalafil facilitates the distal ureteralstone expulsion: a meta-analysis. J Endourol 2017;31:557-63.

7. Becker AJ, Stief CG, Meyer M, Truss MC, Forssmann WG, Jonas U. The effect of thespecific phosphodiesterase-IV-inhibitor rolipram on the ureteral peristalsis of the rabbit invitro and in vivo. J Urol 1998;160:920-5.
8. Buono R, Briganti A, Freschi M, Villa L, Croce GL, Moschini M, et al. Silodosin andtadalafil have synergistic inhibitory effects on nerve-mediated contractions of human andrat isolated prostates. Eur J Pharmacol 2014;744:42-51.

9. Rahman MJ, Faridi MS, Mibang N, Singh RS. Comparing tamsulosin, silodosin versussilodosin plus tadalafil as medical expulsive therapy for lower ureteric stones: Arandomised trial, Arab Journal of Urology,2018;16: 245-249

10. Hari Bahadur KC, Shrestha A, Acharya GB, Basnet RB, Shah AK, ShresthaPM.Tamsulosin versus tadalafil as a medical expulsive therapy for distal ureteral stones: Aprospective randomized study. InvestigClin Urol. 2016; 57:351-356.

11. Jayant K, Agrawal R, Agrawal S. Tamsulosin versus tamsulosin plus tadalafil as medicalexpulsive therapy for lower ureteric stones: a randomized controlled trial. Int J Urol2014;21:1012-5.

12. Kloner R. A., Jackson G., Emmick J. T. et al., "Interaction between the phosphodiesterase 5 inhibitor, tadalafil and 2 alpha-blockers, doxazosin and tamsulosininhealthy normotensive men," Journal of Urology, 2004; 172(5):1935-1940.

13. Kloner R. A., "Cardiovascular effects of the 3 phosphodiesterase- 5 inhibitors approvedfor the treatment of erectile dysfunction," irculation, 2004;110(19):3149-3155.

14. Bechara A., Romano S., Casab'e A. et al., "Comparative efficacy assessment oftamsulosin vs. tamsulosin plus tadalafil in the treatment of LUTS/BPH. Pilot study," Journal of Sexual Medicine,2008;5(9):2170-2178.

15. Rossi M, Roumegue're T. Silodosin in the treatment of benign prostatic hyperplasia. Drug Des DevTher 2010;27(4):291-7. 